\title{
Segmentation of brain MR image using fuzzy local Gaussian mixture model with bias field correction
}

\author{
*Pavan G S , **Nagendra Kumar M,*** Dr.S.Bhargavi \\ IV Sem M.Tech (LSP), Associate Professor, Professor \\ Dept. of ECE, SJCIT Chickballapur
}

\begin{abstract}
Accurate brain tissue segmentation from magnetic resonance (MR) images is an essential step in quantitative brain image analysis. However, due to the existence of noise and intensity in-homogeneity in brain MR images, many segmentation algorithms suffer from limited accuracy. Here, we assume that the local image data within each voxel's neighborhood satisfy the Gaussian mixture model (GMM), and thus propose the fuzzy local GMM (FLGMM) algorithm for automated brain MR image segmentation with bias field correction. This algorithm estimates the segmentation result that maximizes the posterior probability by minimizing an objective energy function, in which a truncated Gaussian kernel function is used to impose the spatial constraint and fuzzy memberships are employed to balance the contribution of each GMM. Our results show that the proposed algorithm can largely overcome the difficulties raised by noise, low contrast, and bias field, and substantially improve the accuracy of brain MR image segmentation.
\end{abstract}

\section{Introduction}

SEGMENTATION of major brain tissues, including gray matter (GM), white matter (WM), and cerebrospinal fluid, from magnetic resonance (MR) images plays an important role in both clinical practice and neuroscience research. However, due to the non-uniform magnetic field or susceptibility effects, brain MR images may contain a smoothly varying bias field, which is also referred to as the intensity in-homogeneity or intensity non-uniformity [1]. As a result, the intensities of the same tissue vary across voxel locations and may lead to segmentation errors. Therefore, bias field correction and segmentation should be interleaved in an iterative process so that they can benefit from each other and yield better results. Many brain MR image segmentation approaches with bias field correction have been proposed in the literature [2]-[12]. Among them, those based on the expectation-maximization (EM) algorithm [2]-[4] and fuzzy C-mean (FCM) clustering [5][12] are the most popular ones.

Pham and Prince. [8] proposed an adaptive FCM (AFCM) algorithm, which incorporates a spatial penalty term into the objective function to enable the estimated membership functions to be spatially smoothed. Ahmed et al. [9] added a neighborhood averaging term to the objective function, and thus developed the biascorrected FCM (BCFCM) algorithm. Liew and Yan. [10] used a B-spline surface to model the bias field and incorporated the spatial continuity constraints into fuzzy clustering algorithms. Li et al. [6] proposed an energyminimization approach to the coherent local intensity clustering (CLIC), with the aim of achieving tissue classification and bias field correction simultaneously. In our previous work [11], [12], we incorporated the global information into the CLIC model to enhance its robustness to the involved control parameters, then we proposed a newly modified possibilistic FCMs clustering algorithm (MPFCM) for bias field estimation and segmentation of brain MR image. Although such modification improves the segmentation accuracy, it also dramatically increases the computational complexity. Various kernel techniques have been used to improve the performance of clustering approaches. Chen and Zhang [13] replaced the original Euclidean distance with a kernel-induced distance and supplemented the objective function with a spatial penalty term, which models the spatial continuity compensation. Yang and Tsai [14] proposed an adaptive Gaussian-kernel-based FCM (GKFCM) algorithm with the spatial bias correction. Liao et al. [15] developed a spatially constrained fast kernel FCM (SFKFCM) clustering algorithm to improve the computational efficiency. However, the clustering performed in a kernel space is generally very time consuming.

Alternatively, brain MR images can be segmented by using the Gaussian mixture model (GMM) [16], where the voxel intensities in each target region are modeled by a Gaussian distribution [17]. The GMM parameters are usually estimated by maximizing the likelihood of the observed image via the EM algorithm [2]. A major drawback of the GMM-EM framework is its lack of taking the spatial information and uncertainty of data into consideration. As a result, it may produce less accurate segmentation results. To remedy this drawback, Greenspan et al. [18] and Blekas et al. [19] incorporated the spatial constraints into the GMM. Tran et al. proposed the fuzzy GMM (FGMM) model to address the uncertainty of data and improve parameter estimation [20]. Zeng et al. [21] developed the type-2 FGMM (T2-FGMM) model for density modeling and classification. 
However, to our knowledge, so far those fuzzy extensions of the GMM-based segmentation algorithm have not been able to overcome the difficulties caused by the intensity in-homogeneity. The conventional GMM implies the stochastic assumption that throughout the image, intensities in the same region are sampled independently from an identical Gaussian distribution. This assumption, however, is invalid for brain MR images due to the existence of the bias field. Based on the fact that the bias field varies very slowly and can be ignored within a small window, in this paper, we assume that the local image data within the neighborhood of each voxel follow the GMM, in which the mean of each Gaussian component is approximated as a tissue dependent constant multiplied by the bias field estimated at this voxel.

Thus, we propose the fuzzy local GMM (FLGMM) algorithm for brain MR image segmentation. The objective function of our algorithm is defined as the integration of the weighted GMM energy functions over the entire image. In the objective function, a truncated Gaussian kernel function is used to impose the spatial constraint, and fuzzy memberships are employed to balance the contribution of each GMM to the segmentation process. The proposed algorithm has been compared to other state-of-the-art segmentation algorithms in both simulated and clinical brain MR images.

\section{A. Bias Field Formulation}

\section{Related work}

The bias field in a brain MR image can be modeled as a multiplicative component of an observed image, as shown in the following:

$$
\mathrm{I}=\mathrm{bJ}+\mathrm{n}
$$

..................1

Where I is the observed image, $\mathrm{J}$ is the true image to be restored, $\mathrm{b}$ is an unknown bias field, and $\mathrm{n}$ is the additive zero-mean Gaussian noise. The goal of bias field correction is to estimate and eliminate the bias field $b$ from the observed image I.

\section{B. Fuzzy C-Mean}

Let $\mathrm{I}=\left\{\mathrm{I}(\mathrm{k}) \in \mathrm{R}^{\mathrm{d}} ; 1 \leq \mathrm{k} \leq \mathrm{n}\right\}$ be a set of d-dimensional image features. The FCM [22] partitions this feature set into c clusters based on minimizing the sum of distances from each feature to every cluster cancroids weighted by its corresponding membership. Let the membership function be $\mathrm{U}=\left\{\mathrm{u}_{\mathrm{i}}(\mathrm{k})\right\} \in \mathrm{R}^{\mathrm{c} \times \mathrm{n}}$, where $\mathrm{u}_{\mathrm{i}}(\mathrm{k}) \in[0,1]$ is the degree of feature $\mathrm{I}(\mathrm{k})$ belonging to cluster $\mathrm{i}$ and follows the constraint $\sum_{i=1}^{c} u i(k)=1$. The quadratic objective function to be minimized is

$$
\mathrm{J}_{\mathrm{FCM}}=\sum_{i=1}^{c} \int u i(k)^{m}|I(k)-v i|^{2} d k \ldots \ldots \ldots .2
$$

Where $v_{i}$ is the cancroids of cluster $i$, and $m \in(1, \infty)$ is the fuzzy coefficient.

\section{Gaussian Mixture Model}

The GMM is a weighted sum of c Gaussian density distributions. With the GMM, the likelihood of the observed data $\mathrm{I}(\mathrm{k})$ is as follows:

$$
\mathrm{P}(\mathrm{I}(\mathrm{k}) \mid \Theta \mathrm{i})=\sum_{i=1}^{c} p_{i} N\left(I(k) \mid \mu_{i} \sum i\right) \ldots \ldots \ldots . .3
$$

Where $\Theta \mathrm{i}=\left\{\mathrm{p}_{\mathrm{i}}, \mu_{\mathrm{i}}, \Sigma_{\mathrm{i}}\right\}$ is the assembly of parameters, and $\mathrm{p}_{\mathrm{i}}$ is the mixing coefficient of $\mathrm{i}^{\text {th }}$ Gaussian component $\mathrm{N}\left(\mathrm{I}(\mathrm{k}) \mid \mu_{\mathrm{i}}, \Sigma \mathrm{i}\right)$ and follows the constraint $\sum_{i=1}^{c} p_{i}=1$. The parameters involved in the GMM are denoted by $\Theta=$ $\left\{\Theta_{\mathrm{i}}, \mathrm{i}=1 \ldots \mathrm{c}\right\}$, and are usually estimated through maximizing the likelihood of observed data via the EM algorithm, as shown in the following:

$\Theta^{*}=\arg \max _{\Theta} \prod_{k=1}^{n}\left[\sum_{i=1}^{c} p_{i} N\left(I(k) \mid \mu_{i} \sum i\right)\right] \ldots 4$

\section{Architectural design for proposed system}

Accurate brain tissue segmentation of MR images has been one of the most important research areas for several years. It is important to have an accurate segmentation of different brain tissue types for various applications such as radiotherapy planning, image-guided interventions, surgical planning by using EEG or fMRI information and brain disease studies such as Alzheimer and MS.

For instance a study on Alzheimer disease carried out on department of radiology in university of California at San Francisco medical center to compare volume of brain tissues in patients with Alzheimer disease and control subjects. They extracted white matter (WM), Grey matter (GM) and Cerebrospinal fluid (CSF) volumes using quantitative tissue segmentation techniques.

Their results showed significant decrease in GM and an increase in the ventricular and sulcal CSF for Alzheimer patients which shows the importance of having an accurate brain tissue segmentation for this study. 
As shown in the figure 1 first we input the MR images and then estimate the bias field using BCFCM and correct the bias field. Second, use the bias corrected MR image as input to the FLGMM algorithm and obtain the segmented output use full for aforementioned brain disease studies such as Alzheimer and MS.

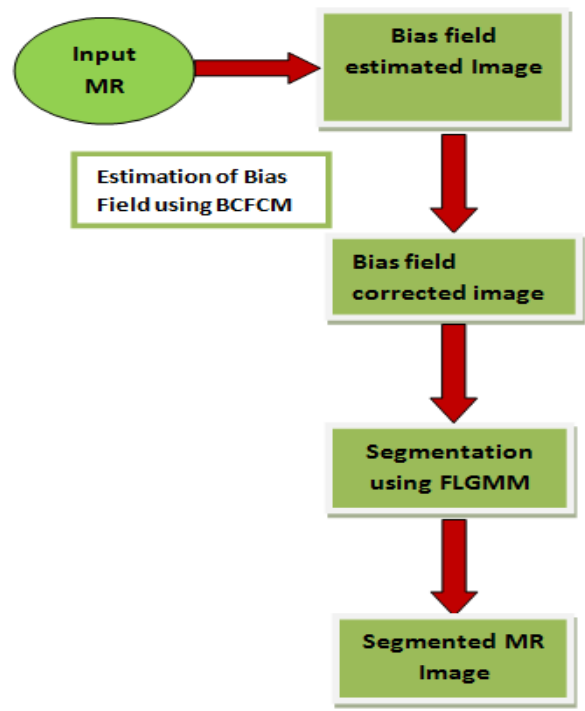

Figure 1: Architectural design for proposed system

\section{Algorithms / Techniques used}

In this section we used two techniques for Accurate brain tissue segmentation by interleaving bias field correction with FLGMM algorithm. One is BCFCM for bias field estimation and correction. Second is FLGMM for segmentation of bias field corrected MR brain images.

\section{A. BCFCM Algorithm}

The BCFCM algorithm for correcting the bias field and segmenting the image into different clusters can be summarized in the following steps.

Step 1.Select initial class prototypes $\left\{v_{i}\right\}_{i=1}^{c}$. Set $\left\{\beta_{k}\right\}_{k=1}^{N}$ to equal and very small values.

Step 2.Update the partition matrix using

$$
u_{i k}^{*}=\frac{1}{\sum_{j=1}^{c}\left(\frac{D_{i k+\frac{\alpha}{N_{R}}} \gamma_{i}}{p\left(D_{j k}+\frac{\alpha}{N_{R}} \gamma_{j}\right)}\right)^{1 / p-1}}
$$

Step 3.The prototypes of the clusters are obtained in the form of weighted averages of the patterns using

$$
v_{i}^{*}=\frac{\sum_{k=1}^{N} u_{i k}^{p}\left(\left(y_{k}-\beta_{k}\right)+\frac{\alpha}{N_{R}} \sum_{y_{r} \in N_{k}}\left(y_{r}-\beta_{r}\right)\right)}{(1+\alpha) \sum_{k=1}^{N} u_{i k}^{p}}
$$

Step 4.Estimate the bias term using

$$
\beta_{k}^{*}=y_{k}-\frac{\sum_{i=1}^{c} u_{i k}^{p} v_{i}}{\sum_{i=1}^{c} u_{i k}^{p}}
$$

Repeat Steps $2-4$ till termination. The termination criterion is as follows:

$$
\left\|V_{\text {new }}-V_{\text {old }}\right\|<\varepsilon
$$

Where $\|$.$\| is the Euclidean norm, \mathrm{V}$ is a vector of cluster centers, and $\varepsilon$ is a small number that can be set by the user.

\section{B. FLGMM Algorithm}

FLGMM for segmentation of bias field corrected MR brain images.

Step 1: Initialization.

Initialize the number of clusters, standard deviation, and neighborhood radius of the truncated Gaussian kernel, cluster centroids, and bias field at each voxel.

Step 2: Updating parameters.

Step 2.1: Updating membership function 


$$
u_{i}(y)=\left(\sum_{i=1}^{c}\left(\frac{d_{i}(I(y))}{d_{j}(I(y))}\right)^{\frac{1}{m-1}}\right)^{-1} .
$$

Step 2.2: Updating covariance matrix

$$
\sum_{i}=\frac{\int u_{i}(y)^{m} k(x-y)\left(\left(I(y)-b(x) v_{i}\right)\left(I(y)-b(x) v_{i}\right)^{T}\right) d y}{\int u_{i}(y)^{m} k(x-y) d y}
$$

Step 2.3: Updating bias field

$$
\mathrm{b}(\mathrm{x})=\frac{\sum_{i=1}^{c} \int k(x-y) u_{i}(y)^{m}\left(I(y)^{T} \sum i(x)^{-1} v_{i}\right) d y}{\sum_{i=1}^{c} \int k(x-y) u_{i}(y)^{m}\left(v_{i}^{T} \sum i(x)^{-1} v_{i}\right) d y}
$$

Step 2.4: Updating mixture weight

$$
p_{i}=\frac{k * u_{i}^{m}}{\sum_{i=1}^{c} k * u_{j}^{m}}
$$

Step 2.5: Updating centroids

$$
v_{i}=\left(\iint u_{i}(y)^{m} k(x-y) b(x)^{2}\left(\sum i(x)^{-1}\right) d x d y\right)^{-1} \times\left(\iint u_{i}(y)^{m} k(x-y) b(x) \cdot\left(\sum i(x)^{-1} I(y)\right) d x d y\right)
$$

Step 3: Checking the termination condition.

If the distance between the newly obtained cluster centers and old ones is less than a user-specified small threshold $\varepsilon$, stop the iteration; otherwise, go to step 2.

\section{Experimental results}

We empirically set the parameters used in our algorithm as follows: the fuzzy factor $m=2$, standard deviation of the kernel function $\tau=4$ and neighborhood radius of the kernel function $\rho=10$. For all the experiments, we initialize the centroids with $k$-means clustering.

\section{A. Segmentation of Synthetic Images}

The first experiment was performed in three synthetic images, which were displayed in the first column of Figure 2. In the first image, the intensities of the star-shaped object and background have the same mean but different variances. The images in the middle and bottom rows were corrupted by intensity in-homogeneity. The intermediate segmentation results obtained by running the proposed algorithm for different numbers of iterations were shown in the second to fourth columns, and the final results obtained after the convergence of our algorithm were shown in the fifth column. It is revealed from Figure 2 that the result gradually improves during the iterative segmentation process.

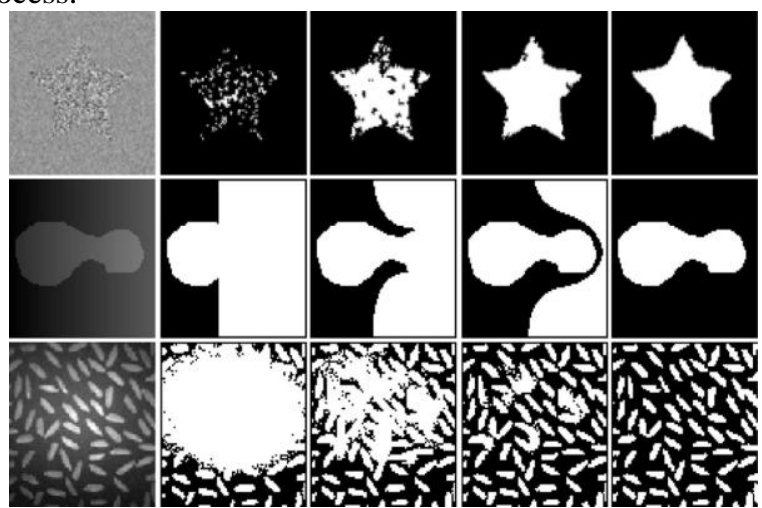

Figure 2. Illustration of (first column) three synthetic image and their (second to forth columns) intermediate and (fifth column) final segmentation results.

\section{B. Segmentation of Brain MR Images}

The second experiment was carried out in 3T- and 7Tweighted clinical brain MR images. Figure 3 shows three 3Tweighted clinical brain MR images that were used in [5], together with the estimated bias fields and segmentation results. 
It is clear from this figure that in spite of the quite obvious bias field and noise in these images, the proposed algorithm can estimate the bias field and achieves satisfactory segmentation results.

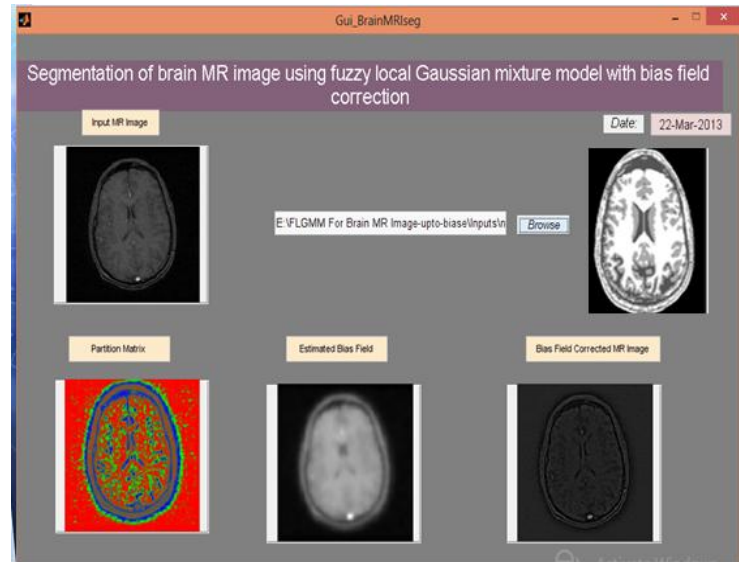

Figure 3. Illustration of (top left column) 3T-weighted brain MR images (Axial View), (bottom left column) the partition matrix, (middle column) estimated bias field, and (bottom right column) bias field corrected image, (top right column) segmented MR image.

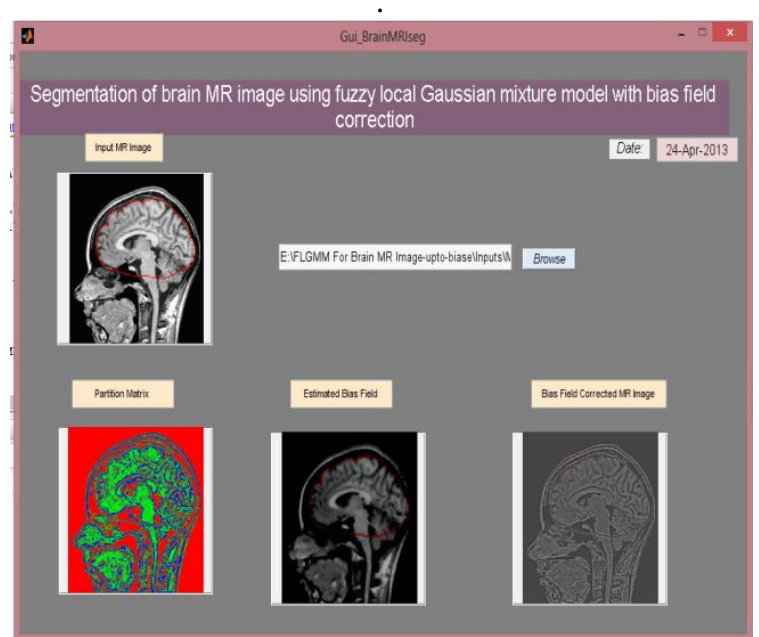

Figure 4. Illustration of (top left column) 7T-weighted brain MR images (sagittal view), (bottom left column) the partition matrix, (middle column) estimated bias field, and (bottom right column) bias field corrected image, (top right column) segmented MR image.

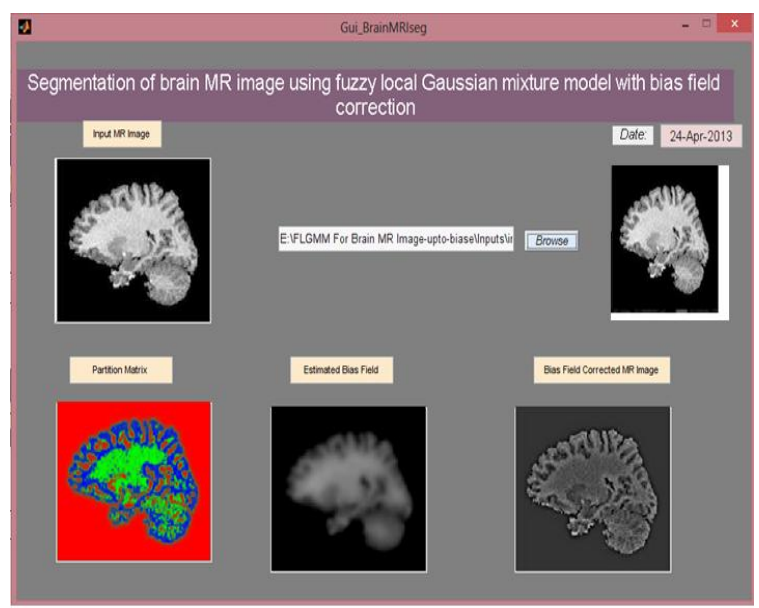

Figure 5. Illustration of (top left column) 3T-weighted brain MR images (Coronal View), (bottom left column) the partition matrix, (middle column) estimated bias field, and (bottom right column) bias field corrected image, (top right column) segmented MR image. 


\section{Quantitative Comparison}

In the third experiment, we quantitatively compared the proposed FLGMM algorithm to eight existing segmentation approaches, including two FCM-based algorithms (AFCM [8] and BCFCM [9]), two kernel FCMbased algorithms (GKFCM [14] and SFKFCM [15]), two EM-based algorithms proposed by Wells et al. [2] and Leemput et al. [3], and two fuzzy membership and local-information-based algorithms (CLIC [6] and MPFCM [12]). To make a fair comparison, all algorithms were initialized by using the $k$-means clustering. The segmentation accuracy was measured by the Jaccard similarity (JS), which is the ratio between intersection and union of the segmented volume $S 1$ and ground truth volume $S 2$

$$
\mathrm{JS}\left(\mathrm{S}_{1}, \mathrm{~S}_{2}\right)=\left|\frac{s_{1} \cap s_{2}}{s_{1} U s_{2}}\right|
$$

The value of JS ranges from 0 to 1 , and a higher JS represents more accurate segmentation.

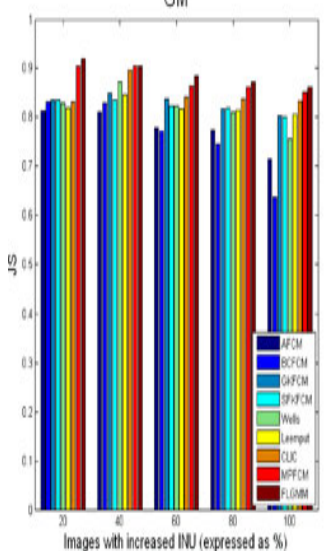

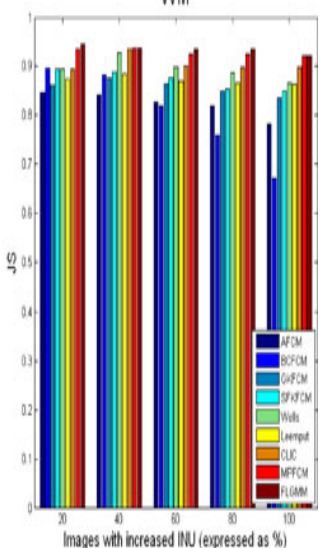

Figure 4. Average JS of (left) GM segmentation and (right) WM segmentation obtained by applying nine segmentation algorithms to simulated brain MR images with increasing levels of intensity in-homogeneity.

\section{Conclusion}

In this paper, we assume that the local image within the neighborhood of each voxel follows the GMM, and thus propose the FLGMM algorithm for brain MR image segmentation. This algorithm uses a truncated Gaussian kernel function to incorporate spatial constraints into local GMMs, and employs the fuzzy membership function to balance the contribution of each GMM to the segmentation process. Our results in both synthetic and clinical images show that the proposed algorithm can largely overcome the difficulties raised by noise, low contrast, and bias fields, and is capable of producing more accurate segmentation results than several state-ofthe-art algorithms.

\section{Future work}

Our future work will focus on reducing its computational complexity and improving its robustness to initialization.

\section{References}

[1] U. Vovk, F. Pernus, and B. Likar, "A review of methods for correction of intensity inhomogeneity in MRI," IEEE Trans.Med. Imag., vol. 26, no. 3, pp. 405-421, Mar. 2007.

[2] W. Wells, E. Grimson, R. Kikinis, and F. Jolesz, “Adaptive segmentation of MRI data," IEEE Trans. Med. Imag., vol. 15, no. 4, pp. 429-442, Apr. 1996

[3] V. Leemput, K. Maes, D. Vandermeulen, and P. Suetens, "Automated model-based bias field correction of MR images of the brain,” IEEE Trans. Med. Imag., vol. 18, no. 10, pp. 885-896, Oct. 1999.

[4] Y. Zhang, M. Brady, and S. Smith, "Segmentation of brain MR images through a hidden Markov random field model and the expectationmaximization algorithm," IEEE Trans.Med. Imag., vol. 20, no. 1, pp. 45- 57, Jan. 2001.

[5] C. Li, C. Gatenby, L. Wang, and J. Gore, "A robust parametric method for bias field estimation and segmentation of MR images," in Proc. IEEE Conf. Comput. Vision Pattern Recog., 2009, pp. 218-223.

[6] C. Li, C. Xu, A. Anderson, and J. Gore, "MRI tissue classification and bias field estimation based on coherent local intensity clustering: A unified energy minimization framework," in Proc. 21st Int. Conf. Inf. Process. Med. Imag., Lecture Notes in Computer Science, 2009, vol. 5636, pp. 288-299.

[7] K. Sikka, N. Sinha, P. K. Singh, and A. K. Mishra, "A fully automated algorithm under modified FCM framework for improved brain MR image segmentation," Magn. Reson. Imag., vol. 27, pp. 994-1004, Jul.2009.

[8] D. Pham and J. Prince, “Adaptive fuzzy segmentation of magnetic resonance images," IEEE Trans.Med. Imag., vol. 18, no. 9, pp. 737-752, Sep.1999.

[9] M. Ahmed, S. Yamany, N. Mohamed, A. Farag, and T.Moriarty, "A modified fuzzy c-means algorithm for bias field estimation and segmentation of MRI data," IEEE Trans. Med. Imag., vol. 21, no. 3, pp. 193-199, Mar. 2002. 
[10] A. Liew and H. Yan, "An adaptive spatial fuzzy clustering algorithm for 3-D MR image segmentation," IEEE Trans. Med. Imag., vol. 22, no. 9, pp. 1063-1075, Sep. 2003.

[11] Z. X. Ji, Q. Chen, Q. S. Sun, D. S. Xia, and P. A. Heng, "MR image segmentation and bias field estimation using coherent local and global intensity clustering," in Proc. 7th Int. Conf. Fuzzy Syst. Knowl. Discov., 2010, vol. 2, pp. 578-582.

[12] Z. X. Ji, Q. S. Sun, and D. S. Xia, "A modified possibilistic fuzzy c-means clustering algorithm for bias field estimation and segmentation of brain MR image," Comput. Med. Imag. Graph., vol. 35, pp. 383-397, Jul.2011.

[13] S. C. Chen and D. Q. Zhang, "Robust image segmentation using FCM with spatial constraints based on new kernel-induced distance measure," IEEE Trans. Syst. Man Cybernet., vol. 34, no. 4, pp. 1907-1916, Apr.2004.

[14] M. S. Yang and H. S. Tsai, "A Gaussian kernel-based fuzzy c-means algorithm with a spatial bias correction," Pattern Recog. Lett., vol. 29, pp. 1713-1725, Sep. 2008. 